\title{
Tracking the Luxury Consumer Online: An Experimental Study on the Effectiveness of Site and Search Retargeting for Luxury Brands in China and the Netherlands: An Abstract
}

\author{
Shubin Yu, Liselot Hudders, and Verolien Cauberghe
}

\begin{abstract}
E-commerce is becoming an unstoppable trend for luxury brands. To boost the online sale, digital marketing strategies have been gradually adopted by luxury markers. However, research on their effectiveness is scarce.

One recent and increasingly popular marketing strategy is retargeting. Retargeting is a form of behavioural targeting in which ads are displayed to consumers based on their surfing behaviours. Site and search retargeting are two specific forms of retargeting. Site retargeting refers to a process in which firms display ads to consumers related to the same website (and product) they recently visited. Search retargeting enables firms to display ads to consumers related to similar products (instead of the exact product and website) they were searching for online (AdRoll 2015). However, the effectiveness of these two types of retargeting is not yet known.

By using a 2 (site vs. search retargeting) $\times 2$ (non-luxury vs. luxury brand) $\times 2$ (China vs. the Netherlands) between-subjects factorial experiment, this study investigates the effectiveness of site vs. search retargeting for luxury brands in the high power distance culture (e.g. the Netherlands) and the low power distance culture (e.g. China). The data is analysed by using the model 12 (moderated mediation) of SPSS macro PROCESS (Hayes 2012).

This study reveals that in the culture of low power distance (e.g. the Netherlands), compared to site retargeting, search retargeting has a positive impact on purchase intention and attitude towards the brand through low perceived intrusiveness. While in the culture of high power distance (e.g. China), compared with site retargeting, search retargeting does not have significant difference in the effectiveness.
\end{abstract}

References Available Upon Request

S. Yu $(\bowtie) \bullet$ L. Hudders $\bullet$ V. Cauberghe

Ghent University, Ghent, Belgium

e-mail: Shubin.Yu@UGent.be; Liselot.Hudders@UGent.be; Veroline.Cauberghe@UGent.be 